\title{
A percepção de mulheres sobre a menstruação: uma questão de solidariedade
}

\author{
Women's perception of menstruation: a question of solidarity \\ La percepción de mujeres sobre la menstruación: una cuestión de solidaridad
}

\author{
Octavio Muniz da Costa Vargens'; Diana da Silva Marinho"l. \\ Alexandra Celento Vasconcellos da Silvall'; Zulmerinda Meira Oliveira ${ }^{\prime V}$
}

\begin{abstract}
RESUMO
Objetivo: descrever a experiência de mulheres em relação à menstruação. Metodologia: estudo qualitativo baseado no método de análise de conteúdo temática. O cenário foi o campus de uma universidade pública no Rio de Janeiro. Participaram 23 mulheres entre 20 e 72 anos de idade. Os dados foram obtidos entre julho/2015 e julho/2016 através da entrevista semiestruturada. Resultados: foi identificada, como tema central/categoria, a questão da solidariedade entre mulheres quando o assunto é menstruação. Emergiram quatro sub-categorias: A conexão das mulheres com a menstruação; A experiência de menstruar afetando umas às outras; A influência e o suporte proveniente das mães e/ou de outras mulheres; e A transferência. Conclusão: a menstruação causa sentimentos ambíguos nas mulheres, resultando em predomínio de sentimentos negativos, como medo e sensação de vergonha. Ao mesmo tempo pode ser percebida como motivo de felicidade e tranquilidade. A solidariedade entre mulheres pode influenciar positivamente a percepção da mulher sobre a menstruação, sendo elemento de empoderamento.
\end{abstract}

Descritores: Menstruação; saúde da mulher; enfermagem obstétrica; apoio social.

\begin{abstract}
Objective: to describe women's experience of menstruation. Methodology: this qualitative study was based on the thematic content analysis method. The setting was a public university campus in Rio de Janeiro. Twenty-three women from 20 to 72 years old participated. Data were obtained between July 2015 and July 2016 by semi-structured interview. Results: the central theme/category was identified as solidarity among women with regard to menstruation. Four subcategories emerged: women's connection with menstruation; one's experience of menstruating affects others; influence and support from mothers and/or other women; and transference. Conclusion: menstruation causes ambiguous feelings in women, with negative feelings, such as fear and a sense of shame, predominating. At the same time, it can be seen as a reason for happiness and tranquility. Solidarity among women is a factor of empowerment, and can influence women's perception of menstruation positively.

Descriptors: Menstruation; women's health; obstetrical nursing; social support.
\end{abstract}

\section{RESUMEN}

Objetivo: describir la experiencia de mujeres en relación con la menstruación. Metodología: estudio cualitativo basado en el método de análisis de contenido temático. El escenario fue el campus de una universidad pública en Río de Janeiro. Participaron 23 mujeres cuya edad estaba entre 20 y 72 años. Los datos se obtuvieron entre julio/2015 y julio/2016 a través de entrevista semiestructurada. Resultados: se identificó, como tema central/categoría, la solidaridad entre mujeres en relación con la menstruación. Surgieron cuatro subcategorías: la conexión de las mujeres con la menstruación; la experiencia de menstruar, afectando unas a las otras; la influencia y el apoyo de las madres y/o de otras mujeres; y la transferencia. Conclusión: la menstruación causa sentimientos ambiguos en las mujeres, resultando en predominio de sentimientos negativos, como el miedo y la sensación de vergüenza. Al mismo tiempo, podría percibirse como un motivo de felicidad y tranquilidad. La solidaridad entre mujeres puede influir positivamente en su percepción sobre la menstruación, siendo un elemento de empoderamiento.

Descriptores: Menstruación; salud de la mujer; enfermería obstétrica; apoyo social.

\section{INTRODUÇÃO}

A menstruação é processo natural que ocorre durante o estágio reprodutivo das mulheres e está presente quando a fertilização do óvulo não ocorre. Historicamente muitas culturas, como por exemplo a judaico-cristã, condenaram o sangue menstrual como algo sujo, mas também como um símbolo de poder feminino, não aceitável para a época ${ }^{1,2}$. Há registros na história, que nas culturas paleolíticas, a mulher é vista como um ser muito poderoso. Menstruar, isto é, apresentar hemorragias mensais, geralmente sem causar complicações, e ser capaz de gerar uma nova vida expressam uma condição da fertilidade feminina².

'Enfermeiro Obstétrico. Doutor, Professor Titular. Universidade do Estado do Rio de Janeiro. Brasil. E-mail: omcvargens@uol.com.br "Enfermeira Obstétrica. Mestre em Enfermagem. Secretaria Municipal de Saúde, Rio de Janeiro. Brasil. E-mail: dmarinho87@gmail.com I'Enfermeira Obstétrica. Mestre em Enfermagem. Universidade do Estado do Rio de Janeiro. Brasil. E-mail: enf.ale.celento@gmail.com IVEnfermeira. Doutora. Doutorado Interinstitucional Universidade do Estado do Rio de Janeiro/ Universidade Estadual do Sudoeste da Bahia. Brasil. E-mail: zulmerindameira@bol.com.br 
A concepção de solidariedade pode ter sentido positivo e esperado, como em uma relação entre casais, amigos, familiares. Também se relaciona à parceria, à confiança e ao apoio nas mais variadas decisões e situações. $O$ sentido designado à atitude de solidariedade, neste estudo, é positivo, pois são demonstrados aspectos como a harmonia, o companheirismo e o entendimento ${ }^{3}$. A menstruação é um fenômeno rotineiro para as mulheres em idade fértil, mas mulheres com experiência negativa são propensas a relatá-la como algo incômodo e insuportável ${ }^{4}$.

Assim, considerando os fatores biológicos, psicológicos e sociais intrínsecos a esse fenômeno, sobre a menstruação, traçou-se como objetivo desta pesquisa descrever a experiência de mulheres em relação à menstruação.

\section{REVISÃO DE LITERATURA}

Baseados nas relações entre homens e mulheres, a sociedade determina um sistema de papéis fundamentados pelo contexto político, econômico e cultural em que se encontram representações do que seja masculino e feminino, havendo ao longo dos tempos a subordinação das mulheres em relação aos homens ${ }^{5}$.

Além das conferências de saúde em nível mundial, um dos destaques ocorreu em 1984, quando o Ministério da Saúde, no Brasil, implementou o Programa de Assistência Integral à Saúde da Mulher (PAISM). Outra conquista relevante - Política Nacional de Atenção Integral à Saúde da Mulher (PNAISM), em 2004, representou um avanço na atenção à saúde da mulher. Com o passar do tempo e novos acontecimentos, aprofundou-se o debate sobre as questões sociais e a consciência feminina ${ }^{6-8}$.

Nesse contexto, encontra-se o gênero, como a construção social do ser masculino e do ser feminino, que traz em sua essência a marca do poder, favorecendo relações desiguais entre homens mulheres ${ }^{6,9}$.

Nesse sentido, os aspectos sociais e culturais da menstruação e sua influência sobre as mulheres, ao longo dos tempos, trazem inquietações. A menstruação é um processo relacionado não somente às mulheres, mas também a seu grupo social, sendo um evento socialmente importante. De acordo com os aspectos socioculturais, cada grupo social possui visão e interpretação distintas sobre a menstruação, que marca a vida da mulher, por ser um período de transição em DOls estágios diferenciados. Há culturas, por exemplo, que levam a menstruação a um alto significado social e comunitário, proporcionando ritos, festividades para as mulheres na fase da menarca. Há outras que excluem as mulheres de seu meio social nesse período. Desse modo, a menstruação, dependendo do ambiente sociocultural da mulher, expressa diferentes crenças, sendo desenvolvidas e transmitidas ao redor desse processo ${ }^{1}$.

No contexto da perspectiva feminista, a mulher, com relação à menstruação, está intimamente ligada às desigualdades de poder e às estruturais que a oprimiram em suas experiências corporais e que, em algumas sociedades, ainda a oprimem. A vergonha menstrual foi identificada como princípio organizador que perpetua a dominação masculina e a subordinação feminina ${ }^{10,11}$.

Porém, contrário ao pensamento hierárquico masculino, tanto a menstruação quanto o nascimento são fontes de autoridade e poder, e fazem com que a mulher esteja à frente do homem na trajetória evolutiva ${ }^{12}$.

A menstruação é frequentemente associada a tabus e mitos que de certa forma influenciam diretamente a relação da mulher com seu meio social, no período menstrual ${ }^{13-15}$. A mulher moderna experimenta mais ciclos menstruais quando comparada às suas precedentes, que vivenciavam a menarca tardiamente, passavam por múltiplas gestações e longos períodos de amamentação e que supriam a menstruação.

A motivação das mulheres parece corresponder a um processo mais complexo, que faz parte do conceito de que a mulher já não é mais obrigada a cumprir com suas funções biológicas. Liberar-se, dessa função biológica, foi uma das grandes conquistas da chamada revolução feminina ${ }^{16,17}$.

Porém, a pressão social para manter a ocultação da menstruação persiste e influencia o comportamento feminino relacionado à saúde ${ }^{18}$. Assim, a menstruação vem sendo vista com ambiguidade: um mal necessário e desagradável que faz parte da natureza da mulher. Ao mesmo tempo, a menstruação tem sido associada à saúde, feminilidade, fertilidade e juventude ${ }^{16,18-20}$.

\section{METOdOLOGIA}

O presente estudo se deu com base no método da análise temática, conforme descrito por Braun e Clarke ${ }^{21}$.

Escolheu-se o método narrativo, que proporciona maior compreensão das experiências vividas pelas mulheres referentes à menstruação. As narrativas são uma forma fundamental de expressão para a comunicação entre os seres humanos, e foram obtidas mediante coleta de dados, em pesquisa qualitativa ${ }^{22}$.

Foram entrevistadas 23 mulheres, entre 20 e 72 anos, a partir da reflexão do tema - menstruação como fenômeno natural. O total de participantes foi determinado pela saturação de informações. Foi empregada a técnica de snowball para encontrar participantes ${ }^{23}$. Como critérios de inclusão, as mulheres deveriam ter ou terem tido ciclos menstruais 
regulares. As mulheres escolheram o local da entrevista, dentro do cenário do estudo, campus de universidade pública, no Rio de Janeiro.

Utilizou-se a entrevista semiestruturada, individual, gravada em equipamento MP3, realizada com base em roteiro próprio, contendo questões de caracterização das informantes e uma questão desencadeadora. Temas adicionais foram apontados para inclusão caso não abordados, espontaneamente, pelas entrevistadas.

Utilizou-se a análise de conteúdo temática, que apresenta seis fases ${ }^{21}$

- Extrair o texto, a partir das entrevistas, através de uma leitura aprofundada das informações, com registro das idéias, familiarizando os dados afins;

- Após o processo; foi gerada uma codificação inicial dos dados de características;

- A busca de temas foi iniciada após a formação de uma lista de diversos códigos;

- Foi analisada a veracidade dos temas. Os dados e os temas deviam estar correlacionados, resultando, assim ,em um mapa temático dessa análise;

- Após a formação do mapa temático, foram definidos e nomeados os temas. Dessa forma, foi possível produzir definições mais simples e até mesmo nomear cada tema específico;

- Por último, a análise final foi realizada para reproduzir o relatório da pesquisa.

O estudo foi realizado em conformidade com a Resolução no 466/2012 do Conselho Nacional de Saúde e aprovado pelo Comitê de Ética em Pesquisa sob parecer $n$ ㅇ 1.507.975 e CCAE $n^{\circ} 55209016.40000 .5282^{24}$. As mulheres receberam informações sobre o objetivo do estudo, e participam de forma voluntária e que a qualquer momento podem retirarse. $O$ consentimento informado foi assinado.

\section{RESULTADOS}

A análise dos dados permitiu identificar como tema central a questão da solidariedade entre mulheres, quando o assunto é menstruação. Assim, esse tema constituiu a principal categoria analítica, cujos desdobramentos/dimensões foram expressos em quatro subcategorias, a saber: A conexão das mulheres com a menstruação; $A$ experiência de menstruar afetando umas às outras; $A$ influência e o suporte proveniente das mães e/ou de outras mulheres; e $A$ transferência.

\section{Uma questão de solidariedade}

Solidariedade, segundo o dicionário Brasileiro da Língua Portuguesa, uma conexão entre duas ou mais pessoas, trocando ideias, sentimentos ou uma situação de um grupo em que são compartilhados atitudes e sentimentos, tornando o grupo mais fortalecido, podendo assim o mesmo resistir às pressões externas ${ }^{3}$.

No contexto da menstruação, a solidariedade está relacionada ao apoio entre as mulheres, à troca de experiências, orientações, confissões e segredos, pois, por ser um assunto de esfera exclusivamente feminina, as mulheres terão melhor manejo para lidar com o tema e, assim, de ajudarem umas às outras diante de qualquer situação. É importante ressaltar que esse apoio ajuda a mulher a se sentir mais segura e empoderada para expor plenamente seus valores, ter um forte senso de pertencimento, reconhecimento e confiança em si mesma.

Eu não fiquei com vergonha, porque minhas primas já tinham ficado menstruadas, já conhecia alguma coisa, minha mãe conversava comigo, foi tranquilo. (E10)

Eu a preparei, disse que chegaria uma idade em que ela ficaria menstruada, tirei as dúvidas dela, mostrei os cuidados que ela deveria ter. (E17)

\section{A conexão das mulheres com a menstruação}

As mulheres, instintivamente ou não, demonstram conexão com a menstruação, entendendo o menstruar como forma de estar intimamente ligada ao seu corpo, à feminilidade, ao "ser mulher". Esta conexão será manifestada em cada mulher de maneira diferente, influenciando em suas vidas.

Ah, é benéfico, saudável, importante para mim... (E7)

É um processo fisiológico normal, significa que eu estou bem. A mulher se sente mais segura para o sexo, mais preparada. (E22)

A menstruação, apesar de ainda ser negligenciada pela modernidade, se fez presente no relato das mulheres como uma conexão às suas origens, pois somente a mulher é o ser capaz de menstruar e assim, há o símbolo de feminilidade, sexualidade e promissora maternidade. 


\section{Como a experiência afeta umas às outras}

A experiência da menstruação é sentida de diversas formas pelas mulheres, pois, para cada mulher o assunto é discutido e vivenciado de formas distintas, dependendo de sua criação, cultura e experiências.

Para algumas mulheres foi uma experiência tranquila, sem grandes preocupações, pois, já haviam sido orientadas, já conheciam o fenômeno menstrual vivenciado por familiares.

Porém, foram abordadas experiências negativas, como a dor, alterações no corpo, mas que posteriormente foram amenizados. Muitos tabus também foram repassados a essas mulheres, resultando, assim, em uma experiência insatisfatória. Em outros casos, esconderam o fato de ter menstruado por medo, vergonha, com o apoio de outras pessoas.

Foi com 12 anos, a minha irmã tinha ficado primeiro que eu e já sabia, minha mãe me ajudou também, não tive grandes barreiras. (E7)

A primeira experiência foi muito ruim, eu fiquei menstruada aos 10 anos, não estava bem preparada, minha mãe também não foi bem preparada e, em consequência ela tinha certos tabus para conversar sobre isso. $A$ experiência foi ruim... (E11)

Eu escondi de todo mundo. Minha mãe só foi saber quando tinha quase um ano. Minha mãe trabalhava e eu ficava com a minha irmã, que tinha 18 anos. Eu tinha 12. Minha cunhada e eu tínhamos a mesma idade. E só ela sabia e foi ela quem me ajudou. Ela comprava os absorventes para mim, dizia que era para ela e eu usava os dela. Depois de muito tempo que a minha mãe viu. Minha mãe chorou porque não tinha necessidade de esconder, mas eu fiquei com vergonha. (E19)

\section{A influência e o suporte proveniente das mães e/ou de outras mulheres}

As narrativas das mulheres apresentaram vários aspectos com relação à solidariedade e a importância da presença de outra mulher quando o assunto é menstruação. A mãe foi citada como uma grande influenciadora e ponto de apoio durante a menarca, tranquilizando, dando orientações, havendo também suporte de amigas e familiares.

Observou-se que algumas mulheres, repensando sobre sua vivência da menarca, reconheceram a má influência dos tabus no comportamento de suas mães quando não atenderam à demanda e às suas expectativas quanto ao processo de menstruação, gerando insatisfações e sentimentos como vergonha e medo.

Foi tranquilo porque eu já havia sido orientada pela minha mãe, e foi só esperar acontecer. Eu não me assustei, não me desesperei, foi tranquilo. (E5)

A primeira vez que ouvi a respeito deste assunto foi com uma amiga. A primeira vez que falei com minha mãe que fiquei menstruada, levei um tapa no rosto. Então, falei com minha amiga e ela disse que eu não poderia transar, ela me passou um anticoncepcional e comecei a usar. (E22)

Mostrou-se extremamente relevante a participação de outra mulher para ajudar a jovem na construção de novo significado da menstruação; o resultado foi surpreendente - as mulheres estão compreendendo a menstruação como natural e pertencente ao corpo feminino e à feminilidade.

\section{A transferência}

É fato que a maneira como a mulher é instruída, na abordagem da menstruação, influenciará na educação dos filhos, ocorrendo a transferência de seus significados e sentimentos à próxima geração, ao longo da vida.

Nessa transferência de orientações, informações são passadas de forma a criar entre as mulheres uma espécie de ligação, uma rede, pois somente elas vivenciam esse fenômeno, compreendem seus medos, dúvidas e, alegrias e, assim, de mãe para filha, de amiga para amiga, enfim entre as mulheres esse tema será passado adiante, pois faz parte de suas vidas.

Ao serem questionadas sobre a mensagem que passarão às suas filhas sobre a menstruação, as respostas foram positivas. Para as mães, é relevante passar as informações e orientações para que tenham boas influências e experiências, pois é algo que faz parte da vida das mulheres.

Eu a preparei, disse que chegaria uma idade em que ela ficaria menstruada, tirei as dúvidas dela, mostrei os cuidados que ela deveria ter e como deveria usar o absorvente, acabou que ela usou errado, mas eu a preparei para isso. Eu conversei e converso sobre tudo, não só sobre menstruação. (E17)

Portanto, constata-se a importância da transferência de orientações e informações para que a mulher seja bem instruída, tenha boas experiências ao longo de sua vida e, assim, ter a capacidade de repassar seus conhecimentos para suas filhas, amigas, enfim outras mulheres que necessitem de orientação. 


\section{DISCUSSÃo}

Como observado nas entrevistas, o tema solidariedade, nesse contexto, está relacionado ao apoio entre as mulheres, à troca de experiências, orientações, confissões, segredos. Há também o aspecto íntimo da menstruação. É um impulso natural não querer anunciá-la, pois as mulheres são educadas a cuidar desse assunto com privacidade. $\mathrm{O}$ sentimento da vergonha por estar menstruada é visto como algo ruim, como se a própria mulher tivesse culpa do que aconteceu com ela, ou seja, estar menstruada. Desse modo, as mulheres expressam de forma negativa os significados da menstruação, pois ainda a veem como algo que deve ser escondido, por ser vergonhoso, estressante e envolver tabus $^{22,25,26}$

Esse comportamento ocorre principalmente entre mãe e filha. A menstruação deve ser enfrentada como algo positivo, sendo uma confirmação do desenvolvimento da mulher e, principalmente, um sinal de saúde ${ }^{22}$.

No entanto, primeiramente, é, através da solidariedade, que a mulher se sentirá mais segura, mais confiante, mais empoderada e, assim, se tornar mais integrada à sociedade ${ }^{23}$.

Entre as mulheres entrevistadas, observou-se que houve uma conexão delas com a questão da menstruação, fazendo-as agirem naturalmente, diante desse sinal de saúde. Além disso, os resultados evidenciaram que a menstruação se fez presente no relato das mulheres como uma conexão às suas origens. A percepção da menstruação vista como um processo natural auxilia as mulheres a relacionarem a menstruação com a feminilidade, a sexualidade, a maternidade, podendo formar um vínculo maior entre mãe e filha ${ }^{16}$.

Observou-se que muitas mulheres identificam a menstruação, como sinal de saúde e normalidade, e que a sua falta é caracterizada como algum tipo de anomalia ${ }^{17}$.

Estudos indicam que as alterações físicas, de humor, cognitivas e comportamentais estão presentes no período menstrual, fazendo a mulher queixar-se de vários sinais e sintomas, compreendidos como distúrbios, cada qual com sua gravidade relacionada a fatores psicológicos, biológicos e ambientais ${ }^{27,29}$.

Porém, há mulheres que não foram orientadas. Aprenderam, na vivência da menstruação, que ser mulher é ser discreta, é ser calada, aceitando o seu corpo com resignação e sem indagações, sendo o mistério um atributo do feminino ${ }^{28}$.

Sobre como a experiência afeta umas às outras, observou-se que a experiência da menstruação é repercutida de diversas formas pelas mulheres, pois, para cada uma o assunto é discutido de uma maneira, sendo orientada ou não, cada uma reage a seu modo. Mostrou-se relevante a participação de outra mulher para ajudar a compreensão da jovem a respeito da menstruação. Algumas mulheres passaram por momentos tranquilos, pois foram orientadas por mãe, irmã. Estranharam inicialmente, mas depois foram devidamente orientadas. Tiveram más experiências, sendo transferidos tabus; não foram orientadas, o que favoreceu a emersão de sentimentos como medos, vergonha; receberam até agressões; mas, também, o apoio de outras pessoas ligadas a elas. E outras mulheres não foram orientadas, porém reagiram naturalmente à situação.

Muitas aprenderam a ser mulheres vivendo e produzindo os seus corpos transpassados por pudores demasiados, conhecendo um cotidiano de segredos, medos e vergonha e, por vezes, repassando também em segredo essa experiência da menstruação. Segundo elas, a experiência que passam com a menstruação é que inicia o processo delas se tornarem mulheres ${ }^{28}$.

Ainda hoje, existem tabus sobre a menstruação, que são transferidos através de gerações pela cultura ancestral histórica. A menstruação apresenta diversos significados simbólicos em várias culturas, como por exemplo, em algumas sociedades indígenas e africanas, em que a menina, após a menarca, é isolada quando passa por um rito de passagem, que irá introduzi-la à vida adulta ${ }^{29}$.

A percepção e as influências se inserem na história de vida das mulheres, incluindo seu meio cultural e vivência. No que diz respeito à menstruação, por ser ainda algo inesperado, muitas mulheres vivenciam seus sinais e sintomas específicos de maneira única e pessoal, pois são alterações vinculadas ao seu contexto biológico, psicológico, nutricional e ambiental, sendo também relevantes o apoio e as orientações dadas pela família ${ }^{14}$.

A influência e o suporte das mães ou outras mulheres como dito anteriormente, são bastante relevantes. A figura materna foi mencionada como a mais influente entre as mulheres. Este apoio, por vezes, é complementado por amigas e familiares, sendo recebido naturalmente.

Para a maioria das mulheres, a mãe é a primeira pessoa a ser cotada para informar sobre a situação, havendo uma resposta e um retorno positivo, na maioria das vezes. Geralmente são as primeiras pessoas que orientam as mulheres, embora, muitas vezes, também de forma negativa contribuam para manter a questão da menstruação em silêncio, impondo restrições aos corpos femininos ${ }^{16,30}$. 
Quando há a ausência da mãe nesse contexto, esse papel geralmente é transferido para a mulher mais próxima de seu círculo de amizades ou familiar, podendo até mesmo o pai atuar nessa função, como observado nos dados, gerando um suporte satisfatório. Em algumas situações, as orientações sobre menstruação fornecidas pelas mães não ofereceram o auxílio necessário às filhas para prepará-las ou simplesmente não foram dadas, assim, apesar do sigilo e negatividade acerca da menarca e da menstruação, as mulheres procuraram buscar informações ouvindo conversas de adultos, através de irmãs mais velhas, amigas ou da escola ${ }^{16}$.

Constatou-se a importância da transferência de orientações, informações para que a mulher seja bem instruída, tenha boas experiências ao longo de sua vida e, assim, ter a capacidade de repassar seus conhecimentos para suas filhas, amigas, enfim para outras mulheres que necessitem de orientação.

A análise evidenciou a importância de se comunicar sobre a menstruação e outros assuntos referentes à sexualidade, especialmente informações sobre as alterações do corpo, cuidados e responsabilidades a serem tomados, gerando assim segurança e tranquilidade à mulher. Destaca-se que esta conversa não é somente realizada por mães, amigas, mas também é abordada na escola por professores. Para algumas jovens, a escola é a única fonte de informação, pois não foram orientadas pelos pais, e os professores se tornam a principal fonte de conhecimento ${ }^{16}$.

A enfermagem pode exercer papel fundamental para o empoderamento feminino, em sua lida com o fenômeno da menstruação ao estimular e potencializar a solidariedade entre mulheres. Atuando como mediadora de práticas educativas centradas no acolhimento e promoção da segurança poderá auxiliar a mulher a se fortalecer e a trazer um sentido/significado positivo da menstruação em sua vida ${ }^{31}$.

\section{CONCLUSÃO}

Conclui-se que diversos aspectos podem influenciar a percepção individual das mulheres sobre a menstruação e como a solidariedade em relação a esse fenômeno é relevante para elas.

A menstruação não deve ser vista somente como um fator natural e de saúde, mas também como social, pois é através da cultura e das representações sociais que esse processo é construído.

Além disso, cada mulher vive a sua cultura do seu jeito, mesmo que tenha sido orientada e educada, para vivenciar papéis representativos do feminino. Cada uma tem sua experiência aliada, juntamente, às representações culturais do seu grupo, como por exemplo, mães, vizinhas, irmãs e colegas de trabalho; dessa forma apropria-se de variados modos de vivenciar o corpo e, havendo a solidariedade, estará mais fortalecida.

Observou-se que a menstruação é causadora de sentimentos ambíguos nas mulheres, resultando em um predomínio de sentimentos negativos, como medo, tristeza, e sensação de vergonha. Mas, também houve sentimentos de felicidade, tranquilidade e satisfação.

Os relatos apontaram que, como a sexualidade, a menstruação é tema pouco discutido. Pertence somente a elas, tornando-as aliadas quanto à questão.

À enfermagem caberá ajudar a mulher a ressignificar o fenômeno da menstruação, como algo natural, possibilitando que ela assuma a menstruação como símbolo do feminino e instrumento de empoderamento. Isto constitui ação importante do cuidado de enfermagem, por valorizar seu cunho libertador.

\section{REFERÊNCIAS}

1. Gómez-Sánchez PI, Pardo- Mora YY, Hernández-Aguirre HP, Jiménez-Robayo SP, Pardo-Lugo JC. Menstruation in history. Invest. Educ. Enferm. [Internet]. 2012; [cited 2019 Feb 05];30(3): 371-7. Available from: http://www.scielo.org.co/scielo.php?script=sci_arttext\&pid=S0120-53072012000300010

2. Cordovil D. Women's Power in Wicca: an Analysis of the "Women's Circle". Estudos Feministas. [Internet]. 2015; [cited 2019 Feb 05]; 23(2): 431-49. DOI: http://dx.doi.org/10.1590/0104-026X2015v23n2p431.

3. Significado de solidariedade. Michaelis. Dicionário Brasileiro da Língua Portuguesa. [Internet]. 2018 [aprox.1 tela]. [cited 2019 Feb 05]. Available from: https://michaelis.uol.com.br/moderno-portugues/busca/portugues-brasileiro/solidariedade

4. Mcpherson M, Korfine L. Menstruation across time: menarche, menstrual attitudes, experiences, and behaviors. Women's Health issues [Internet]. 2004; [cited 2019 Feb 05]; 14(6):193-200. DOI: http://dx.doi.org/10.1016/j.whi.2004.08.006.

5. Cabral FB, Ressel LB, Landerdahl MC. Nursing appointment: a strategy of approaching to the pregnant woman in the perspective of the gender. Esc. Anna Nery [Internet]. 2005; [cited 2017 May 08]; 9(3): 459-65. DOI: http://dx.doi.org/10.1590/S141481452005000300014

6. Ministério da Saúde (BR). Secretaria Especial de Políticas para as Mulheres. Plano Nacional de Políticas para as Mulheres. Brasília (DF): Ministério da Saúde; 2004.

7. Ramalho KS, Silva ST, Lima, SM, Santos, M A. Política de saúde da mulher à integralidade: efetividade ou possibilidade? Cad. de Graduação - Ciências Hum. e Sociais Fits. [Internet] 2012; [cited 2019 Feb 05]; 1(1): 11-22. Available from: http://periodicos.set.edu.br/index.php/fitshumanas/article/view/462/198 
8. Ministério da Saúde (Br). Secretaria de Atenção à Saúde. Departamento de Ações Programáticas Estratégicas. Manual de atenção à mulher no climatério/menopausa. Brasília (DF): Ministério da Saúde; 2008; [cited 2019 Feb 05]. Available from: http://portal.saude.gov.br/portal/arquivos/pdf/manual_climaterio.pdf.

9. Bandeira LM. Violência de gênero: a construção de um campo teórico e de investigação. Soc. estado [Internet]. 2014; [cited 2019 Feb 05]; 29(2): 449-69. DOI: http//dx.doi.org/10.1590/S0102-69922014000200008

10. Moloney S. How menstrual shame affects birth. Women Birth [Internet]. 2010 [cited 2019 Feb 05]; 23(4):153-9. DOI: http://doi.org/10.1016/j.wombi.2010.03.001

11. Tan DA, Haththotuwa R, Fraser IS. Cultural aspects and mythologies surrounding menstruation and abnormal uterine bleeding. Best Pract. Res. Clin. Obstet. Gynaecol. [Internet] 2017; [cited 2019 Feb 05]; 40:121-33. DOI: http://dx.doi.org/10.1016/j.bpobgyn.2016.09.015

12. Kunzler ML. Participação da mulher na política representativa. Fazendo gênero 8 - Corpo, Violência e Poder. [Internet] 2008; [cited 2019 Feb 05]; Available from: http://www.fazendogenero.ufsc.br/8/sts/ST29/Maria_Laci_Kunzler_29.pdf

13. Luz AMH, Berni NIO, Selli L. Myths and taboos of motherhood: focusing on the health-disease process. Rev. Bras. Enferm. [Internet]. 2007; [cited 2019 Feb 05]; 60(1):42-8. DOI: http://dx.doi.org/10.1590/S0034-71672007000100008.

14. Ratti CR, Azzellini EC, Barrense H, Grohmann R. O tabu da menstruação reforçado pelas propagandas de absorvente. In: Congresso brasileiro de ciências da comunicação - Intercom; 2015; Rio de Janeiro, Brasil. Sociedade Brasileira de Estudos Interdisciplinares da Comunicação, Rio de Janeiro; 2015.

15. Omari OA, Razeq NMA, Fooladi MM. Experience of menarche among jordanian adolescent girls: an interpretive phenomenological analysis. Pediatr. Adolesc. Gyneco., [Internet] 2016; [cited 2019 Feb 05]; 129: 246-51. DOI: http://dx.doi.org/10.1016/j.jpag.2015.09.005

16. Ribeiro CP, Hardy E, Hebling EM. Preference of Brazilian women regarding menstrual changes. Rev. Bras. Ginecol. Obstet. [Internet]. 2007; [cited 2019 Feb 05]; 29(2):74-9. DOI: http://dx.doi.org/10.1590/S0100-72032007000200003.

17. Bocchino S. Salud mental de la mujer: Síntomas y trastornos premenstraules. Rev Psiquiatr Urug [Internet]. 2004; [cited 2019 Feb 05]; 68(1):78-89. Available from: http://www.spu.org.uy/revista/jun2004/06_ed_med.pdf

18. O'Flynn, N. Menstrual symptoms: the importance of social factors in women's experiences. Br. J. Gen. Pract. [Internet] 2006; [cited 2019 Feb 05]; 56(533):950-7. Available from: https://www.ncbi.nlm.nih.gov/pmc/articles/PMC1934056/

19. Valadares GC, Ferreira LV, Correa FH, Romano-Silva MA. Premenstrual dysphoric disorder review - concept, history, epidemiology and etiology. Rev. psiquiatr. clín. [Internet]. 2006; [cited 2019 Feb 05]; 33(3):117-23. DOI: http://dx.doi.org/10.1590/s0101-60832006000300001.

20. Silva, LDC, Mamede MV. Unveiling the senses and meanings of the climacteric in coronary women. Ciênc. Cuid. Saúde [Internet]. 2017; [cited 2019 Feb 05]; 16 (2): 1-8. Available from: http://www.periodicos.uem.br/ojs/index.php/CiencCuidSaude/article/view/31719

21. Braun V, Clarke V. Using thematic analysis in psychology. Qualitative Research in Psychology. [Internet] 2006; [cited 2019 Feb 05]; 3(2): 77-101. Available from: http://eprints.uwe.ac.uk/11735

22. Sousa MGS, Cabral CLO. A narrativa como opção metodológica de pesquisa e formação de professores. Revista Horizontes [Internet]. 2015 [cited 2019 Feb 05]; 33 (2): 149-58. Available from: http://eprints.uwe.ac.uk/11735

23. Baldin N, Munhoz EMB. Snowball (bola de neve): uma técnica metodológica para pesquisa em educação ambiental comunitária. In: Congresso Nacional de Educação - EDUCERE, 2011; Curitiba, Paraná. Curitiba (PR): Pontifícia Universidade Católica do Paraná; 2011. p.329-41.

24. Conselho Nacional de Saúde. Brasil. Resolução $n^{\circ} 466$, de 12 de dezembro de 2012. Dispõe sobre a pesquisa envolvendo seres humanos. Diário Oficial da União, Brasília, DF, 13 jun. 2013. Seção 1, p. 59-62.

25. Diogenes MAR. Dismenorreia: a vivência expressa por adolescentes. Cogitare Enferm. [Internet]. 2000 [cited 2019 Feb 05]; 5(2): 15-21. DOI: http://dx.doi.org/10.5380/ce.v5i2.44879

26. Fahs B. Genital panics: constructing the vagina in women's qualitative narratives about pubic hair, menstrual sex, and vaginal self-image. Body Image. 2014 [cited 2019 Feb 05]; 11(3): 210-18. DOI: http://dx.doi.org/10.1016/j.bodyim.2014.03.002

27. Zola CJ, Schiavo RA. Um olhar psicanalítico sobre a menstruação. Instituto Municipal de Ensino Superior de São Manuel ‘Dro Aldo Castaldi' IMESS [Internet]. 2013 [cited 2019 Feb 05]; 1-4. Available from:

http://www.imessm.edu.br/wpcontent/uploads/2014/07/UM-OLHAR-PSICANAL\%C3\%8DTICO-SOBRE-AMENSTRUA\%C3\%87\%C3\%830.pdf

28. Fáveri $M$, Venson AM. Entre vergonhas e silêncios, o corpo segredado. Práticas e representações que mulheres produzem na experiência da menstruação. Anos 90 [Internet]. 2007 [cited 2019 Feb 05]; 14 (25) :65-97. Available from: http://seer.ufrgs.br/anos90/article/viewFile/5403/3060

29. Brêtas JRS, Tadini AC, Freitas MJD, Goellner MB. Meaning of menarche according to adolescents. Acta paul. enferm. [Internet]. 2012 [cited 2018 apr 26]; 25(2): 249-55. DOI: http://dx.doi.org/10.1590/S0103-21002012000200015

30. Jackson TE, Falmagne RJ. Women wearing white: discourses of menstruation and the experience of menarche. Feminism \& Psych. [Internet]. 2012 [cited 2019 Feb 05] 23 (3): 379-98. DOI: https://dx.doi.org/10.1177/0959353512473812

31. Costa R, Santos I, Progianti J. Obstetric nurses' skills as mediators of the educational process: sociopoetic study. Rev. enferm. UERJ. [Internet] 2016. [cited 2018 Feb 05]; 24(4):e18864. DOI: https://dx.doi.org/10.12957/reuerj.2016.18864 